\title{
Cancer Biobank
}

National Cancer Institute

\section{Source}

National Cancer Institute. Cancer Biobank. NCI Thesaurus. Code C15863.

A repository of biospecimens - including tissue samples, fluids such as blood, serum and urine, and molecular derivatives such as DNA, RNA and proteins pertaining to cancer research. The repository has string ent guidelines regarding the standardized collection, handling, storage and documentation of biological specimens. These guidelines enable an extensive system for specimen storage that allows for the accurate establishment of disease incidences over long periods of time. 